\title{
Effect of Carbohydrate Intake on Maximal Power Output and Cognitive Performances
}

\author{
Laura Pomportes ${ }^{1,2, *}$, Jeanick Brisswalter ${ }^{1}$, Arnaud Hays ${ }^{3}$ and Karen Davranche ${ }^{4, *}$ \\ 1 Université Cote d'Azur, Laboratoire Motricité Humaine Expertise Sport Santé, Nice 06205, France; \\ brisswalter@unice.fr \\ 2 CREPS PACA, Aix en Provence 13098, France \\ 3 Aix-Marseille Université, CNRS, ISM, Marseille 13288, France; arnaud.hays@univ-amu.fr \\ 4 Aix-Marseille Université, CNRS, LPC UMR 7290, Marseille 13331, France \\ * Correspondence: laurapomportes@hotmail.fr (L.P.); karen.davranche@univ-amu.fr (K.D.); \\ Tel.: +33-413-551-135 (K.D.)
}

Academic Editor: Beat Knechtle

Received: 30 July 2016; Accepted: 26 September 2016; Published: 9 October 2016

\begin{abstract}
The present study aimed to assess the beneficial effect of acute carbohydrate $(7 \% \mathrm{CHO})$ intake on muscular and cognitive performances. Seventeen high levels athletes in explosive sports (fencing and squash) participated in a randomized, double-blind study consisting in series of 6 sprints (5s) with a passive recovery (25s) followed by $15 \mathrm{~min}$ submaximal cycling after either maltodextrine and fructose $(\mathrm{CHO})$ or placebo $(\mathrm{Pl})$ intake. Cognitive performances were assessed before and after sprint exercise using a simple reaction time (SRT) task at rest, a visual scanning task (VS) and a Go/Nogo task (GNG) during a submaximal cycling exercise. Results showed a beneficial effect of exercise on VS task on both conditions (Pl: $-283 \mathrm{~ms}$; $\mathrm{CHO}:-423 \mathrm{~ms}$ ) and on SRT only during $\mathrm{CHO}$ condition $(-26 \mathrm{~ms})$. In the $\mathrm{CHO}$ condition, SRT was faster after exercise whereas no effect of exercise was observed in the Pl condition. According to a qualitative statistical method, a most likely and likely positive effect of $\mathrm{CHO}$ was respectively observed on peak power $(+4 \%)$ and tiredness $(-23 \%)$ when compared to Pl. Furthermore, a very likely positive effect of $\mathrm{CHO}$ was observed on SRT $(-8 \%)$ and a likely positive effect on visual scanning $(-6 \%)$ and Go/Nogo tasks $(-4 \%)$ without any change in accuracy. In conclusion acute ingestion of $250 \mathrm{~mL}$ of $\mathrm{CHO}, 60 \mathrm{~min}$ and $30 \mathrm{~min}$ before exercise, improve peak power output, decrease muscular tiredness and speed up information processing and visual detection without changing accuracy.
\end{abstract}

Keywords: $\mathrm{CHO}$; maximal muscular performance; information processing

\section{Introduction}

Classically, the ergogenic effects of carbohydrate $(\mathrm{CHO})$ ingestion on physical performance have been demonstrated in the field of sports nutrition research. Indeed, as early as 1925, Gordon et al. [1] reported that ingestion of candy by runners during a marathon prevented hypoglycemia and improved race times when compared with no sugar. The "metabolic response" of CHO feeding allows maintaining plasma glucose concentration and high rates of carbohydrate oxidation, sparing hepatic glycogen, delaying muscle glycogen depletion and preventing hypoglycemia [2]. The beneficial effect of $\mathrm{CHO}$ supplementation during exercise also seems to affect the central nervous system (CNS) by increasing or maintaining the substrate delivery to the brain and avoiding hypoglycemia, which is known to impair brain function and cognitive performances [3]. Further evidences suggest that $\mathrm{CHO}$ may have a "non-metabolic" central effect. According to the "Central Fatigue hypothesis" proposed by Newholme et al. in 1987 [4], tryptophan (which is an essential amino acid and a precursor of serotonin) is associated with central nervous system fatigue during prolonged exercise. It appears that $\mathrm{CHO}$ 
feeding decreases the amount of tryptophan crossing the blood-brain barrier and consequently leads to lower serotonin concentrations in the brain $[3,5]$.

The positive effect of $\mathrm{CHO}$ feedings on performance has been mainly observed during prolonged exercise ( $>1 \mathrm{~h}$ ) (for review see [6]). Endurance exercise has been associated with a decrease in $\mathrm{CHO}$ availability that includes significant glycogen reduction, low blood glucose, and elevations in free fatty acids. These phenomena can be avoided by $\mathrm{CHO}$ feedings. In intermittent sports that required brief high-intermittent exercise, more mixed results have been reported. Most studies report an enhancement of intermittent high-intensity exercise capacity (measured by the duration to fatigue and distance covered) with $\mathrm{CHO}$ supplementation before and during exercise (for review see [7]). Mixed results are reported on other aspects of intermittent sport performance such as sprinting, jumping, skill, change of direction speed, and cognitive function (for review see [8]). For short duration exercise the effect of $\mathrm{CHO}$ feeding is unlikely explained by a metabolic response but more by an ergogenic effect highlighted using mouth rinsing. In a study employing a Loughborough Intermittent Shuttle Test (LIST) protocol (mimicking the demands of soccer and similar team sports), Dorlin and Earnest [9] found no effect of mouth rinsing $6.4 \%$ maltodextrin, in comparison with a mouth rinsing placebo. By contrast, using a self-selected pacing LIST protocol, Rollo et al. [10] reported improved sprint performance with mouth rinsing $10 \%$ maltodextrin compared to a placebo. It is likely that $\mathrm{CHO}$ mouth rinsing exerts a positive effect through a central action mediated by receptors in the mouth or gastro-intestinal tract, improving motor drive or motivation [11-14]. Gant et al. [11] first showed that $\mathrm{CHO}$ in the mouth immediately increases the excitability of the cortico-motor pathway, prior to ingestion. More recently, using a qualitative approach, De Pauw et al. [12] showed an increased activity within the orbitofrontal cortex during maltodextrine mouth rinse, but did not report any change on response speed in a Stroop task. The improvement in exercise performance observed when $\mathrm{CHO}$ is present in the mouth can be explained by an activation of regions involved in reward and motor control (primary taste cortex and dorsolateral prefrontal cortex) [13] and by increasing the primary sensorimotor cortex, which enhances activation of neural networks involved in sensory perception [14]. The effect of $\mathrm{CHO}$ ingestion on sprint performance, however, remains unclear. In a review on this topic, Baker et al. indicate that most of the studies report no benefit of $\mathrm{CHO}$ ingestion on sprint performance [8]. However some of them indicate that $\mathrm{CHO}$ ingestion could improve performance. For example, Gant et al. [11] reported enhanced sprint performance during a 60-min LIST protocol when soccer players ingest a $6.2 \% \mathrm{CHO}$ solution, and Ali et al. [15] reported similar benefit with a $6.4 \%$ CHO solution during a 90-min LIST protocol. Additionally, Lee et al. [16] showed that CHO intake prior to exercise provided a significant benefit on repetition sprint performance in female athletes. Finally, Welsh et al. [17] and Winnick et al. [18] reported improvement in sprint performance when basketball players respectively intake $80 \mathrm{~g}$ of carbohydrate per hour $(\mathrm{g} / \mathrm{h})$ and $40 \mathrm{~g} / \mathrm{h}$.

Intermittent court sports like squash or fencing are characterized by intermittent bursts of high-intensity exercise and require the execution of complex sport-specific skills and cognitive tasks over a prolonged period of time of several times per day [8]. Athletes have to simultaneously perform very intense short exercises and make decisions under strong temporal pressure. The physiological effects of exercise on cognitive performance have been well documented $[19,20]$. According to the different meta-analyses on exercise and cognition, the presence or absence of a beneficial effect of exercise on cognitive performance depends on the nature of the cognitive task but also on exercise intensity and duration [21]. A positive effect of acute moderate exercise is generally explained by an activation of the CNS, with the assumption that exercise-induced physical arousal leads to a narrowing of attentional focus [22], whereas a heavy exercise or prolonged exercise to exhaustion leads to a decrease in cognitive performance [23]. Within this framework, it has been suggested that nutritional supplementation could help to limit central fatigue and increase or maintain cognitive abilities [24]. For example, cognitive performance improvement has been observed during exercise when $\mathrm{CHO}$ was ingested before or during exercise [22,25-27]. Collardeau et al. 2001 [22] showed that consumption of a 5.5\% CHO electrolyte solution before and during a 100-min run improved the 
reaction time (RT) of triathletes at the end of exercise. Bottoms et al. [25] also reported that ingestion of a 6.4\% CHO drink improved RT performances during a simulated squash play. Lieberman et al. [26] found that supplemental $\mathrm{CHO}$ beverages (12\%) enhance vigilance and mood during sustained aerobic exercise (road march and run), and Utter et al. [27] reported that $\mathrm{CHO}$ availability attenuates exertion perceptions during the last stages of $2.5 \mathrm{~h}$ high-intensity cycling and running exercise.

The current state of the art showed that $\mathrm{CHO}$ feedings could improve both physical and cognitive performances; however, the effect of $\mathrm{CHO}$ supplementation on cognitive functions has been essentially assessed during endurance exercises. The present study aimed to investigate the effect of $\mathrm{CHO}$ supplementation on intermittent sport performance, more particularly on sprint and cognitive performances, during a protocol involving brief high-intermittent exercise in high-level athletes in intermittent sports (e.g., squash and fencing).

\section{Methods}

\subsection{Participants}

Seventeen high-level squash and fencing athletes (Table 1) (7 females and 10 males) volunteered in the study. Participants were used to training on a cycle ergometer and cycling as a normal part of their daily training program. The experimental procedures were explained to the subjects and a written informed consent was provided and signed prior to inclusion. The present study was approved by the University Ethics Committee.

Table 1. Anthropometrical and physiological characteristics of participants.

\begin{tabular}{cccc}
\hline Variables & $\begin{array}{c}\text { All } \\
\text { Standard Error }\end{array}$ & Squash Athletes & Fencing Athletes \\
& 17 & 8 & 9 \\
Sample size & $19.1 \pm 1$ & $20.8 \pm 2$ & $17.3 \pm 0.3$ \\
Age (years) & $170.6 \pm 2$ & $174.5 \pm 4$ & $167.1 \pm 3$ \\
Height $(\mathrm{cm})$ & $63.9 \pm 3$ & $67.1 \pm 5$ & $61.1 \pm 2$ \\
Body mass $(\mathrm{kg})$ & $15.3 \pm 1$ & $11.9 \pm 1$ & $18.4 \pm 2$ \\
Body fat $(\%)$ & $48.5 \pm 2$ & $50.7 \pm 1$ & $47.5 \pm 4$ \\
$\mathrm{VO}_{2 \max }\left(\mathrm{mL} \cdot \mathrm{min}^{-1} \cdot \mathrm{kg}^{-1}\right)$ & $200.8 \pm 2$ & $199.2 \pm 2$ & $202 \pm 3$ \\
$\mathrm{Heart} \mathrm{rate}_{\max }\left(\mathrm{batt} \cdot \mathrm{min}^{-1}\right)$ & $12.7 \pm 1$ & $12.7 \pm 1$ & $12.8 \pm 1$ \\
Blood lactate post test $\left(\mathrm{mmol} \cdot \mathrm{L}^{-1}\right)$ & $14.5 \pm 0.4$ & $16.2 \pm 0.4$ & $14.5 \pm 0.5$ \\
Maximal aerobic speed $\left(\mathrm{km} \cdot \mathrm{h}^{-1}\right)$ & & & \\
\hline
\end{tabular}

\subsection{Design}

\subsubsection{Preliminary Session}

One week before the experimental sessions, a preliminary testing was performed to collect anthropometric and physiological characteristics. Body weight was measured to $0.1 \mathrm{~kg}$ using a standard beam balance. Height was measured to $0.5 \mathrm{~cm}$ using a Holtain wall mounted stadiometer. In all subjects, four skinfold thickness (biceps, triceps, subscapular, suprailiac) were measured in triplicate, by the same trained observer. Measurements were made on the right-hand side of the body using a Holtain caliper. Body fat was calculated using the Siri equation. Maximal oxygen uptake $\left(\mathrm{VO}_{2 \max }\right)$ was determined during a field maximal incremental exercise according to the protocol proposed by Léger and Boucher [28]. The speed of the last fully completed step corresponds to the maximal aerobic speed. During the test, oxygen uptake $\left(\mathrm{VO}_{2}\right)$, carbon dioxide uptake $\left(\mathrm{VCO}_{2}\right)$, minute ventilation $(\mathrm{VE})$ and the respiratory exchange ratio (RER) were continuously recorded using a breath-by-breath analyzer (K4b2, Cosmed ${ }^{\circledR}$, Rome, Italy) which was calibrated prior to each test. Heart rate was collected using a monitor (Polar RS800). Before and 3 min after running sessions, blood lactate concentrations were measured (LactatePro, Arkray Factory Inc., Shiga, Japan). 
The target heart rate of the experimental sessions was determined from a competition simulation respectively in squash ( 5 players) and fencing ( 5 players) against a similar level opponent. For the squash simulation, 5 games of 11 points ( 1 for each players lasting between 8 and $13 \mathrm{~min}$ ) were recorded and analyzed, whereas for fencing athletes, 5 matches of 3 min were recorded. From these records, the relative intensity was set at $80 \%$ of $\mathrm{HR}_{\max }$ for fencing athletes and $85 \% \mathrm{FC}_{\max }$ for squash athletes.

\subsubsection{Familiarization Session}

Seventy-two hours prior to the first experimental session, subjects underwent a familiarization session with the repeated sprint effort protocol on a cycle ergometer and with the following cognitive tasks (CT): simple reaction time (SRT), visual scanning and Go/Nogo tasks (TAP software 2.3 version 3, P. Zimmermann and B. Fimm). For CT, the learning criteria was achieved when response variability was equal or less than $10 \%$ compared to the preceding cognitive performance.

\subsubsection{Experimental Session}

Each subject participated in two counterbalanced experimental sessions separated by 1 week at the same time of the day. Subjects were instructed to keep a food diary during the 2 days prior the first session and to replicate this diet before each session. Subjects were required to refrain from alcohol, caffeine, drugs, and nutritional supplements during the $48 \mathrm{~h}$ prior the sessions. The last meal was taken at least $3 \mathrm{~h}$ prior the start of the experimental session. No food or drinks intake (except water) was allowed between the last meal and the experimental session. The subjects were not used to regularly drinking nutritional supplementation during training or competitions. They were instructed to control their time and hours of sleep the 2 nights preceding the experimental sessions. Light trainings were permitted in the morning of the experimental sessions whereas physical training (including endurance or resistance training) or high-intensity technical sessions were not allowed. Subjects were required to maintain the same training program every week during the experimental protocol.

During each experimental session, subjects ingested $60 \mathrm{~min}$ (Ing1) and $30 \mathrm{~min}$ (Ing2) before exercise either $250 \mathrm{~mL}$ of a $7 \%$ carbohydrate complex (CHO: fructose and maltodextrine, Isoxan Sport,) or placebo ( $\mathrm{Pl}, 250 \mathrm{~mL}$ tap water added with orange sugarless syrup). Immediately after the second ingestion subjects performed the first cognitive tests block (i.e., CT). Then, subjects warmed up (standardized warm up) and after 3 min recovery performed, they performed a repeated sprint test in order to assess maximal power output. Immediately after this test, subjects completed one block of SRT. Finally, they performed a 15 min submaximal cycling exercise at $80 \%$ and $85 \% \mathrm{HR}_{\max }$, respectively for fencing and squash athletes, during which they performed the visual scanning and Go/Nogo tasks (Figure 1).

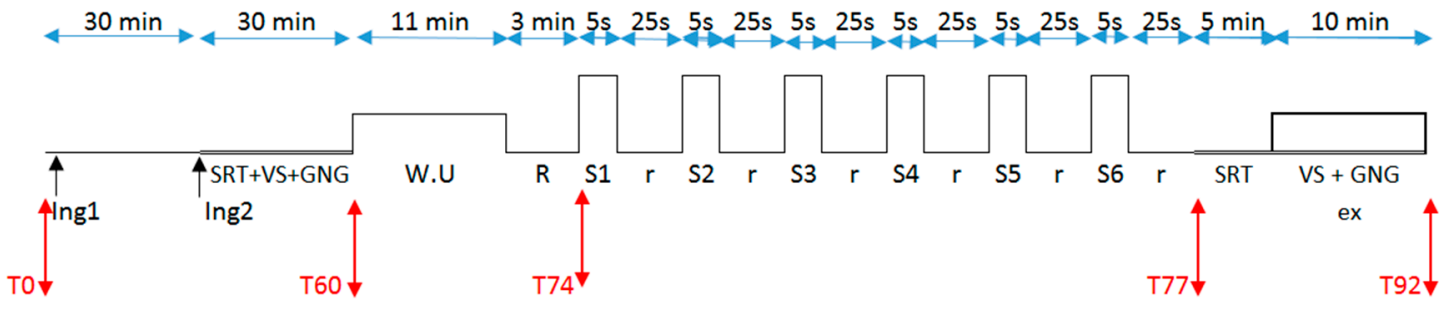

Figure 1. Overview of the experimental design. Notes: Ing = ingestion, W.U = warm up, R = rest 3 min, $\mathrm{S} 1$ to $\mathrm{S} 6=$ sprints $5 \mathrm{~s}, \mathrm{r}=25 \mathrm{~s}$ rest, $\mathrm{SRT}=$ simple reaction time, VS = visual scanning, $\mathrm{GNG}=\mathrm{Go} / \mathrm{Nogo}$ tasks, ex = cycling exercise, $\mathrm{T}=$ time (minutes).

\subsection{Repeated Sprint Test}

Maximal power output was assessed during the repeated sprint test on a Wattbike cycle (Wattbike Pro, Nottingham, UK). This ergometer has been designed to simulate "real" cycling with a suitable power output range (0 to 3760 watts) for short-duration, high-intensity testing, or training, 
and has been previously validated to assess sprint performance in cyclists [29]. After a specific warm up for sprint performance, subjects performed $6 \times 5 \mathrm{~s}$ sprints (S1 to S6), with a $25 \mathrm{~s}$ passive recovery between each sprint (the resistance was set at the minimum, resistance 1). Maximal power output was recorded for each sprint, and tiredness (\%) was calculated using the difference between maximal power output for the best and the last sprint.

\subsection{Cognitive Tasks (CT)}

During the two experimental sessions, subjects were comfortably seated on the cycle ergometer facing a computer screen 1 meter away. Two joysticks were installed on the ergocycle. Participants were required to complete the $\mathrm{CT}$ that consisted in a series of 3 cognitive tasks (i.e., SRT, visual scanning and Go/Nogo tasks) before and after the series of 6 sprints. The SRT was performed at rest immediately after the sprints, and the visual scanning and Go/Nogo tasks were performed while cycling about $15 \mathrm{~min}$ at $80 \%$ and $85 \%$ HRmax respectively for fencing and squash athletes.

\subsubsection{Simple Reaction Time (SRT)}

Simple reaction time is a perceptual-motor task indicative of the efficiency of the individual's ability to process information. In the SRT task, subjects were asked to respond as quickly as possible to the visual known stimuli by pressing the button of a joystick with their dominant hand. The SRT task duration was about $3 \mathrm{~min} 30 \mathrm{~s}$.

\subsubsection{Visual Scanning Task}

Visual scanning task (TAP 2.3 software) is an attention/perceptual task, during which subjects had to search a display for a given stimulus. The display was a matrix on a row-by-row or column-by-column basis $(5 \times 5$ stimuli). Subjects were instructed to press one key response as soon as possible when they detected the target stimulus, and to press another key response when the target stimulus was absent. The visual scanning task duration was about $5 \mathrm{~min}$.

\subsubsection{Go/Nogo Task}

The Go/Nogo task evaluates inhibitory control. Subjects were instructed to respond to stimuli and withhold the response to other stimuli. In the present version of the task (TAP 2.3 software), five squares with different patterns appeared on the screen. Two of these squares were defined as target stimuli. Subjects were instructed to press a key response as soon as possible when a target stimulus was presented, and withhold their response when a non-pertinent stimulus appeared. The Go/Nogo task duration was about $3 \mathrm{~min}$.

\subsection{Statistical Analysis}

Results were analyzed using consecutively quantitative and qualitative methods. All data are expressed as the mean \pm standard error. For sprint performance (i.e., mean peak power and tiredness of 6 sprints), difference between conditions was assessed using a paired t-test. For cognitive performances, a repeated measures ANOVA (condition $\times$ period) was performed. Post-hoc Newman-Keuls analyses were conducted for all significant effects. Significance was set at $p<0.05$ for all analyses. We also reported probabilistic magnitude-based inferences for all variables data using methods described by Hopkins et al. [30], which has been applied in several recent nutritional studies [14]. To compare within-trial changes between trials, we used a modified statistical spreadsheet. This spreadsheet calculates the between-trial standardized differences or effect sizes (ES, 90\% confidence interval [CI]) using the pooled standard deviation. If the chance of benefit or harm were both $>5 \%$ the true effect was reported as unclear. Otherwise, chances of benefit or harm were assessed as follows: $<1 \%$, almost certainly not; $1 \%-5 \%$, very unlikely: $5 \%-25 \%$, unlikely; $25 \%-75 \%$, possible; $75 \%-95 \%$, likely; $95 \%-99 \%$, very likely; $>99 \%$, most likely [5]. 


\section{Results}

\subsection{Sprint Performance}

There were no significant differences between conditions ( $\mathrm{CHO}$ vs. $\mathrm{Pl}$ ) on peak power and tiredness. Magnitude-based inferences showed that $\mathrm{CHO}$ ingestion has a most likely positive effect on peak power (99\%, CI: 9.4/48.8 watts) (Figure 2A,B). Mean peak power was $1043 \pm 255$ watts in $\mathrm{CHO}$ and $1007 \pm 218$ watts in Pl. Furthermore a likely positive effect (77\%, CI: 0.8/5.2\%) was observed on tiredness (Figure 2C) with a lower decrease of peak power among sprints when compared to Pl (respectively for $\mathrm{CHO}$ and $\mathrm{Pl}:-11 \% \pm 4.9 \%$ vs. $-13 \% \pm 5.8 \%$ ).
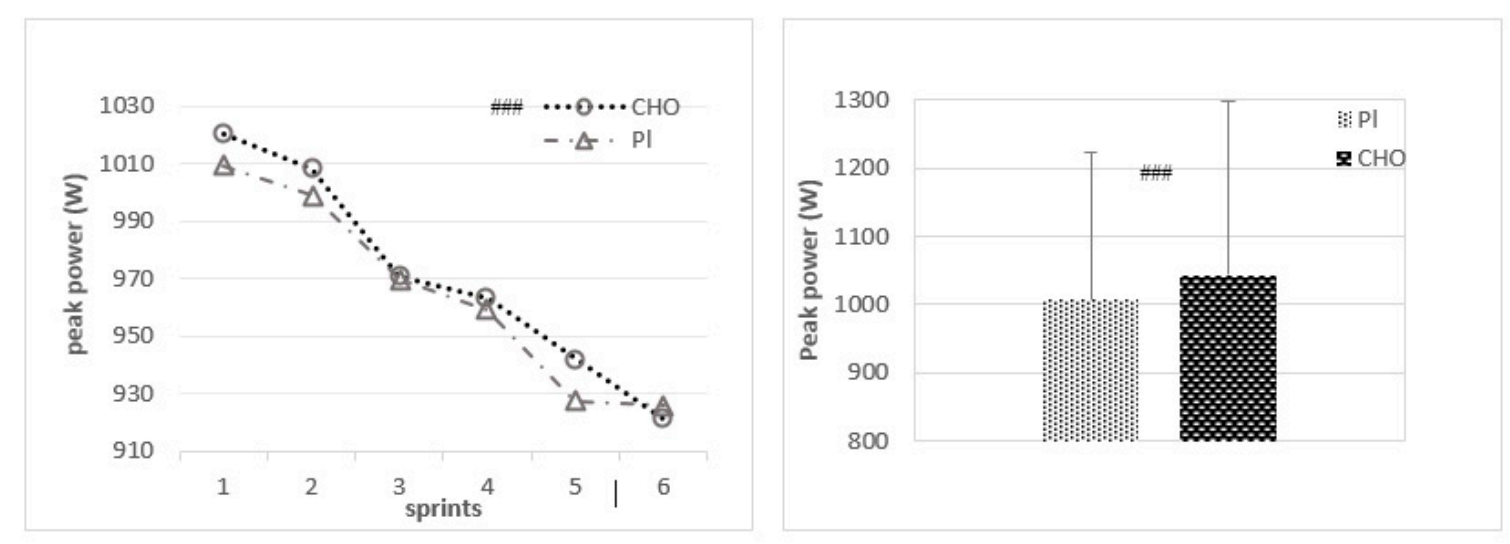

A B

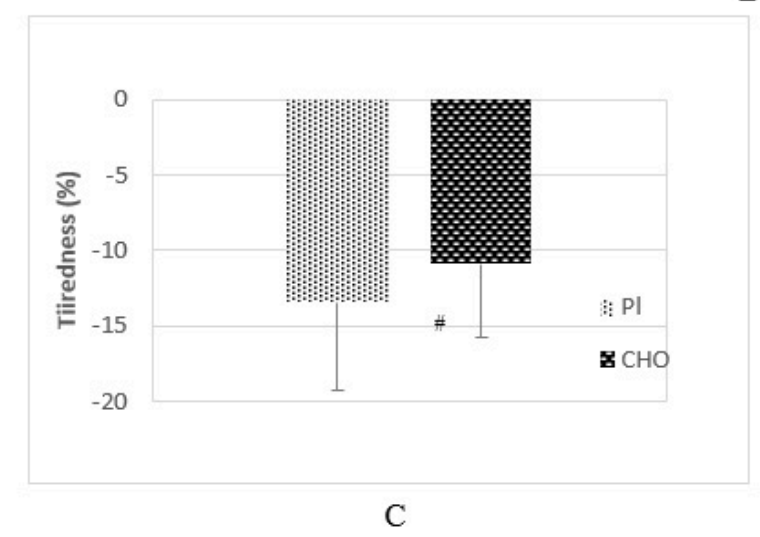

Figure 2. Evolution of peak power with sprint (A); peak power (B) and tiredness (C) with placebo (Pl) and carbohydrate $(\mathrm{CHO})$ ingestion (mean \pm standard error). Notes: \# when a qualitative statistics shown an effect compared with Pl condition: \# likely effect, \#\# very likely effect and \#\#\# most likely effect.

\subsection{Cognitive Performance}

An overview of the results for cognitive performance is shown on Table 2. 
Table 2. Cognitive performance prior and after sprints repetition for $\mathrm{Pl}$ and $\mathrm{CHO}$ ingestion.

\begin{tabular}{|c|c|c|c|c|}
\hline \multirow[b]{2}{*}{ Variables } & \multicolumn{2}{|c|}{ Prior sprints } & \multicolumn{2}{|c|}{ After sprints } \\
\hline & $\underset{\text { Mean } \pm \text { Standard Error }}{\text { Ml }}$ & $\mathrm{CHO}$ & P1 & $\mathrm{CHO}$ \\
\hline SRT & & & \multicolumn{2}{|c|}{ At rest } \\
\hline $\mathrm{RT}(\mathrm{ms})$ & $222.5 \pm 9$ & $229.0 \pm 8$ & $220.4 \pm 8^{\$}$ & $203.1 \pm 5^{\$, £, \#, *}$ \\
\hline $\mathrm{SD}(\mathrm{ms})$ & $88.6 \pm 22$ & $67.0 \pm 15^{£}$ & $74.8 \pm 12$ & $108.9 \pm 22^{£}$ \\
\hline Visual Scanning & & & \multicolumn{2}{|c|}{ During cycling exercise } \\
\hline $\mathrm{RT}(\mathrm{ms})$ & $2010.3 \pm 107$ & $2054.1 \pm 133$ & $1726.8 \pm 73^{\$, *}$ & $1630.6 \pm 76^{\$, E, *}$ \\
\hline $\mathrm{SD}(\mathrm{ms})$ & $867.3 \pm 78$ & $829.0 \pm 75^{£}$ & $689.6 \pm 49^{\$, *}$ & $665.2 \pm 60^{\$, *}$ \\
\hline Tiredness (\%) & $3.8 \pm 3$ & $5.5 \pm 2$ & $1.5 \pm 2^{\$}$ & $-0.4 \pm 2^{\$}$ \\
\hline Errors (\%) & $4.8 \pm 1$ & $5.9 \pm 2^{£}$ & $7.1 \pm 1^{\$, *}$ & $7.8 \pm 1^{\$, *}$ \\
\hline Go/Nogo & & & \multicolumn{2}{|c|}{ During cycling exercise } \\
\hline $\mathrm{RT}(\mathrm{ms})$ & $444.1 \pm 17$ & $453.1 \pm 13$ & $453.1 \pm 18^{\$}$ & $435.4 \pm 12^{\$, E}$ \\
\hline $\mathrm{SD}(\mathrm{ms})$ & $84.2 \pm 7$ & $88.9 \pm 9^{£}$ & $90.1 \pm 9$ & $79.1 \pm 8^{£}$ \\
\hline Errors (\%) & $6.3 \pm 2$ & $5.3 \pm 1$ & $2.6 \pm 1^{\$}$ & $4.9 \pm 1^{\$}$ \\
\hline
\end{tabular}

Notes: * quantitative statistics shown an exercise effect (Pre sprints $\mathrm{Pl} / \mathrm{CHO}$ vs. Post sprints $\mathrm{Pl} / \mathrm{CHO}$ );

\# quantitative statistics shown a nutritional effect $\left(\mathrm{Pl}_{\text {pre/post }}\right.$ vs. $\left.\mathrm{CHO}_{\text {pre/post }}\right) ;{ }^{\$}$ qualitative statistics shown an exercise effect (Pre sprints $\mathrm{Pl} / \mathrm{CHO}$ vs. Post sprints $\mathrm{Pl} / \mathrm{CHO}$ ); ${ }^{£}$ qualitative statistics shown a nutritional effect $\left(\mathrm{Pl}_{\text {pre/post }}\right.$ vs. $\left.\mathrm{CHO}_{\text {pre/post }}\right)$.

\subsubsection{Simple Reaction Time (SRT)}

A significant interaction effect between exercise and condition was observed on mean RT $\left(\mathrm{F}_{(1,16)}=6.95, \mathrm{p}=0.002, \eta_{\mathrm{p}}^{2}=0.30\right)$. In the $\mathrm{CHO}$ condition, RT was faster after exercise $(-11 \%)$ compared to pre-exercise and faster than $\mathrm{Pl}$ post-exercise $(-7.9 \%)$. No effect of exercise was observed in the $\mathrm{Pl}$ condition $(-1 \%)$. Magnitude-based inferences showed that the effect of exercise is likely positive ( $93 \%$, CI: $-43.3 / 8.7 \mathrm{~ms}$ ) on RT (i.e., faster RT) on both conditions, and the effect of CHO ingestion, compared to $\mathrm{Pl}$, is likely positive $(96 \%, \mathrm{CI}$ : $-15.5 / 3.7 \mathrm{~ms})$ on RT performance decrease after exercise.

No significant effect of nutritional supplementation and/or exercise was found on standard deviation (respectively $\mathrm{F}_{(1,16)}=0.11, \mathrm{p}=0.074, \eta_{\mathrm{p}}{ }^{2}=0.007$ and $\mathrm{F}_{(1,16)}=1.05, \mathrm{p}=0.032, \eta_{\mathrm{p}}{ }^{2}=0.06$ ). Magnitude-based inferences showed that the effect of $\mathrm{CHO}$ ingestion, compared to $\mathrm{Pl}$, is likely positive ( $78 \%$ CI: $-23 / 11 \mathrm{~ms}$ ) on SD before sprints and likely negative $(90 \%, \mathrm{CI}:-8 / 16 \mathrm{~ms}$ ) on SD (i.e., increase in SD) after sprints indicating a higher variability in response after exercise.

\subsubsection{Visual Scanning}

Results showed a main effect of exercise $\left(\mathrm{F}_{(1,15)}=24.78, \mathrm{p}=0.000, \eta_{\mathrm{p}}{ }^{2}=0.62\right)$ on RT during visual scanning regardless of the conditions. Reaction time was faster after exercise for Pl $(-14 \%)$ and $\mathrm{CHO}(-21 \%)(\mathrm{p}<0.001)$. Magnitude-based inferences showed that the effect of exercise is most likely positive $(100 \%, \mathrm{CI}:-148 /-67 \mathrm{~ms})$ in both conditions. Furthermore, $\mathrm{CHO}$ ingestion has a likely positive effect $(86 \%, \mathrm{CI}:-12.2 /-0.2)$ on RT when compared to Pl during exercise.

Results showed a main effect of exercise $\left(\mathrm{F}_{(1,15)}=20.58, \mathrm{p}=0.000, \eta_{\mathrm{p}}{ }^{2}=0.58\right)$ on SD in both conditions. A significant decrease of the SD was observed after exercise for $\mathrm{Pl}(-20 \%)$ and $\mathrm{CHO}$ $(-20 \%)$ conditions. Magnitude-based inferences showed the effect of exercise is most likely positive $(100 \%$, CI: $-220 /-105 \mathrm{~ms})$ in both conditions. Furthermore, CHO ingestion has a likely positive effect $(96 \%,-117 / 26 \mathrm{~ms})$ on SD before sprints when compared to Pl.

No effect of nutritional supplementation and exercise was observed on tiredness (respectively $\mathrm{F}_{(1,15)}=0.00, \mathrm{p}=0.098, \eta_{\mathrm{p}}{ }^{2}=0.00$ and $\left.\mathrm{F}_{(1,15)}=2.72, \mathrm{p}=0.012, \eta_{\mathrm{p}}{ }^{2}=0.15\right)$. Magnitude-based inferences indicated that exercise has a likely positive effect $(87 \%, \mathrm{CI}:-12.5 / 0.5 \%)$ on tiredness (decrease), regardless of the nutritional supplementation used. 
Results showed a main negative effect of exercise on errors $\left(\mathrm{F}_{(1,16)}=5.05, \mathrm{p}=0.004, \eta_{\mathrm{p}}{ }^{2}=0.240\right)$ (increase of errors). No effect of nutritional supplementation was observed $\left(\mathrm{F}_{(1,16)}=1.78, \mathrm{p}=0.021\right.$, $\left.\eta_{\mathrm{p}}{ }^{2}=0.10\right)$. Magnitude based inferences showed that the effect of exercise is likely negative $(79 \%, \mathrm{CI}$ : $0.2 / 3.9 \%$ ) on accuracy in both conditions whereas $\mathrm{CHO}$ ingestion is likely negative ( $94 \%, \mathrm{CI}: 0.2 / 2.2 \%$ ) on accuracy when compared to Pl before sprints.

\subsubsection{Go/Nogo}

No effect from nutritional supplementation and exercise was observed on mean RT (respectively $\mathrm{F}_{(1,16)}=0.26, \mathrm{p}=0.062, \eta_{\mathrm{p}}{ }^{2}=0.02$ and $\left.\mathrm{F}_{(1,16)}=0.14, \mathrm{p}=0.072, \eta_{\mathrm{p}}{ }^{2}=0.01\right)$. Magnitude-based inferences showed that exercise has a likely positive effect $(70 \%, \mathrm{CI}:-43.8 / 8.2 \mathrm{~ms})$. Furthermore, the effect of $\mathrm{CHO}$ ingestion is likely positive (82\%, CI: - 11.8/0.2) on RT compared with Pl condition during exercise.

No effect from nutritional supplementation and exercise was found on $\mathrm{SD}$ (respectively $\mathrm{F}_{(1,16)}=0.13$, $\mathrm{p}=0.072, \eta_{\mathrm{p}}{ }^{2}=0.01$ and $\left.\mathrm{F}_{(1,16)}=0.19, \mathrm{p}=0.067, \eta_{\mathrm{p}}{ }^{2}=0.01\right)$. Magnitude-based inferences showed that, when compared to $\mathrm{Pl}, \mathrm{CHO}$ ingestion effect is likely negative $(94 \%, \mathrm{CI}:-6.1 / 17.9 \mathrm{~ms})$ on SD pre-sprint and likely positive during exercise ( $84 \%$, CI: $-26.6 / 6.2 \mathrm{~ms})$.

No effect from nutritional supplementation and exercise was observed on errors (respectively $\mathrm{F}_{(1,16)}=0.27, \mathrm{p}=0.061, \eta_{\mathrm{p}}{ }^{2}=0.02$ and $\left.\mathrm{F}_{(1,16)}=2.63, \mathrm{p}=0.013, \eta_{\mathrm{p}}{ }^{2}=0.14\right)$. Magnitude-based inferences showed that exercise has a likely positive effect $(90 \%, \mathrm{CI}:-2.6 / 0.7 \%)$ on accuracy on both conditions.

\section{Discussion}

The present study aimed to investigate the effect of $\mathrm{CHO}$ ingestion on muscular and cognitive performances using multiple sprints exercise with incomplete recovery $(6 \times 5 \mathrm{~s}$ sprints with $25 \mathrm{~s}$ of passive recovery). Using a qualitative approach [30], the present findings indicated that $\mathrm{CHO}$ ingestion enhances both sprint and cognitive performances. Carbohydrate supplementation increased maximal power output and reduced muscular tiredness compared to $\mathrm{Pl}$ ingestion. The results also demonstrated a positive effect of intermittent heavy exercise on all cognitive tasks, manifested by faster information processing and a better efficiency of attention/perception and inhibitory control processes. When compared to $\mathrm{Pl}$, magnitude-based inferences showed that $\mathrm{CHO}$ ingestion induced a noticeable positive effect on SRT performances after exercise and on Go/Nogo and visual scanning performances during exercise.

In the scientific literature, few studies examined the effect of $\mathrm{CHO}$ ingestion on sprint performance. Moreover, it is difficult to interpret current results considering their disparity. The complex nature of intermittent sports as well as many methodological differences are most likely responsible for such discrepancy. The improvement in muscular performances observed in the present study argues in favor of an ergogenic effect of $\mathrm{CHO}$, in line with previous results indicating an increase in maximal power output after $\mathrm{CHO}$ ingestion [16] or mouth rinse [31,32]. Lee et al. [16] have observed that $\mathrm{CHO}$ ingestion has a small but significant effect on repetition sprint exercise performance in female athletes during 10 sets of $5 \times 4 \mathrm{~s}$ with $20 \mathrm{~s}$ recovery. More recent studies have used mouth rinsing to test the central nervous system hypothesis of $\mathrm{CHO}$ effect. For example, Beaven et al. [31] have reported an increase in power production during a repetition of $5 \times 6$-s cycling sprints interspersed with $24 \mathrm{~s}$ of active recovery in males cyclists. Recently, Philips et al. [32] have investigated the influence of serial administration of $\mathrm{CHO}$ mouth rinse on cycling performance, nausea scales and perceived exertion during a $30 \mathrm{~s}$ cycle sprint in physically active males. Using a qualitative approach, they reported a likely benefit of the $\mathrm{CHO}$ mouth rinse on maximal power output during a cycle sprint, especially during the first $5 \mathrm{~s}$ of the sprint, without any effects on fatigue index or perceived exertion. The present results confirm the positive effect of $\mathrm{CHO}$ supplementation on peak power and power maintenance among sprints on a highly trained population. In the field of elite sport, these findings open a new perspective on performance optimization strategy in order to cope with fatigue.

The second main finding of this study is the effect of $\mathrm{CHO}$ ingestion on cognitive functioning after a series of shorter but high intermittent exercises. It is now widely accepted that exercise has 
a positive effect on cognitive performance and leads to increased arousal [33]. Exercise facilitates multiple cognitive performances and, under certain conditions, enhances responses speed and response accuracy, and facilitates cognitive processes. Most studies focused on the effects of moderate intensity exercise, but results on cognition during heavy exercise are fairly equivocal (for review see [34]). It appears that a positive effect is mainly observed after or during aerobic exercise with a minimum duration of approximately 20 to $60 \mathrm{~min}$ [33]. The hypothesis supporting these results is that exercise acts as a stressor that induces changes in arousal level and as a result impacts cognitive functioning. In the acute exercise-cognition interaction, the effect of exercise seems to be highly correlated with the plasma adrenaline (epinephrine) and noradrenaline (norepinephrine) threshold [35]. For example, it has been reported a link between a high-level of blood adrenaline that could be associated with changes in the central nervous system and improvement in cognitive performance [36]. More recently, Dietrich and Audiffren [23] proposed a neurocognitive model to account for the psychological consequences of acute exercise. This model called reticular-activating hypofrontality model, predicts that exercise engages arousal mechanisms in the reticular-activating system and disengages the higher-order functions of the prefrontal cortex with fatigue. However, this model is still matter of debate [37,38]. Recent studies suggest that cognitive control is extremely robust and appears not to be affected by the heavy exercise. For example, Davranche et al. [37] investigated whether workload intensity modulates exercise-induced effect on RT performances. Fourteen participants performed a Simon task while cycling $20 \mathrm{~min}$ at a light (first ventilatory threshold, VT $-20 \%$ ), moderate (VT1) or very hard (VT $+20 \%$ ) level of exercise. After 15 min of cycling, RT was faster than during the first 5 min of exercise, and any signs of worsening on RT or on accuracy have been reported during heavy exercise. More interestingly, the inhibitory control was fully efficient from the beginning to the end of exercise, regardless of the workload intensity. The results of the present study are in line with these findings, since a positive effect of exercise has been observed on response speed for SRT, visual scanning and Go/Nogo performances after sprint repetition, and suggest that a series of shorter heavy intermittent exercises could enhance cognitive functioning.

The effect of $\mathrm{CHO}$ supplementation on cognitive function during exercise has been occasionally assessed, but mainly during endurance exercise. Lieberman et al., 2002 [26] reported that administration of supplemental carbohydrate significantly enhances vigilance and mood during sustained physical activity and interspersed rest. Collardeau et al. [22] have shown that, when compared to placebo, drinking a 5.5\% carbohydrate solution improves complex cognitive performance during a two-hour run performed at $75 \% \mathrm{VO}_{2 \max }$ by well-trained triathletes. These studies suggest that there is an increase in resource allocation with $\mathrm{CHO}$ ingestion during prolonged exercise. Carbohydrate ingestion during exercise possibly leads to a decrease in mental load associated with the perception of effort (RPE) and consequently lead to a higher involvement in attentional resources during the sustained attention tasks [39]. Indeed the role of CHO ingestion on RPE has been widely studied and an improvement in RPE (lower scores) is classically reported.

There are limited data regarding the effect of $\mathrm{CHO}$ ingestion on cognitive function in intermittent sports and the results are conflicting. To date, Bottoms et al. [25] are the only authors who have reported an improvement in visual $\mathrm{RT}$ after $\mathrm{CHO}$ intake during short duration exercise performed by squash players. Despite the fact that their protocol is far closer to the demands of squash play than the present one, the present findings are in line and support the beneficial effect of $\mathrm{CHO}$ ingestion on sprint performance. Welsh et al. [17] and Winnick et al. [18] tested the effect of CHO ingestion with basketball players on an intermittent high-intensity protocol. The authors reported a benefit of $\mathrm{CHO}$ ingestion on exercise capacity, but no effects on attention and executive control (e.g., Stroop task) were found.

The present results showed that exercise improves cognitive performances. Furthermore, a positive additional effect of $\mathrm{CHO}$ ingestion has been found on information processing during the SRT task and on attention/perceptual performance during the visual scanning. However, no significant changes have been observed on inhibitory control during the Go/Nogo task. One specificity of our 
design is that the SRT task was performed after sprint, at rest, contrary to VS and GNG which were assessed after sprints but during a cycling exercise. According to Lambourne and Tomporowski [33], deterioration of perceptual responses and SRT has been shown when cognitive tasks are performed in double task. The effect of double tasks (exercise + cognitive task) seems to be more important on simple reaction time than other cognitive tasks.

Two mechanisms have emerged to explain the ergogenic effect of $\mathrm{CHO}$ ingestion. The first mechanism, which appears during prolonged exercise lasting more than $1.5 \mathrm{~h}$, is a metabolic effect of $\mathrm{CHO}$ oxidation when skeletal muscle and liver glycogen stores are a limiting factor [40]. For shorter exercise, lasting one hour or less, the explanatory mechanism is of central origin in response to exposure of $\mathrm{CHO}$ in the oral cavity. Considering the brief duration of the physical task used in this protocol, the facilitating effect cannot be explained by a metabolic effect. We assume that during ingestion, $\mathrm{CHO}$ may exert its effects on exercise through a central action, improving motor drive or motivation, mediated by receptors in the mouth or GI tract [40]. The mouth receptors that are sensitive to $\mathrm{CHO}$ could have activated specific brain areas, like the orbitofrontal cortex, and elicit beneficial effects on muscular and cognitive performances [10].

Numerous studies over the past decade emphasized the potential ergogenic effects of dietary constituents on cognitive performance (for review see [3]). In line with the present findings, it has been suggested that nutritional supplementation such as caffeine [41-43], guarana [44,45], tyrosine in a warm environment [46], branched-chain amino acids [47], and nitrate [48] could help to limit central fatigue and increase or maintain cognitive abilities. These findings open a new perspective in terms of performance optimization strategy to cope with fatigue in the field of elite sport. However, more studies are required to complement these results.

The main limitation of the present study is that participants took part in the study in a post-pradial state but no blood glucose baseline levels were measured before experimental session. The fact that the initial carbohydrate status of the athlete was not measured does not question the effects of $\mathrm{CHO}$ observed in this study, but makes the generalization of the present findings difficult. Indeed, the blood level of glucose is an important factor since $\mathrm{CHO}$ ingestion seems to have a greater impact on performance under circumstances eliciting hypoglycemia. The influence of a $\mathrm{CHO}$ intake on performance may be reduced when participants are fed [49,50], therefore the ergogenic effect of $\mathrm{CHO}$ ingestion may have been larger if participants had fasted longer than three hours. However several observations indicate that the nutritional status of participants prior testing is not a definitive regulator of the effect of carbohydrate. Whitham and McKinney [51] found no effect of CHO MR on 60-min time trial performance despite an overnight fast, whereas Pottier et al. [52] found a benefit when a high-CHO meal was ingested two hours prior to testing. While the present study involved a relatively small sample size due to the specificity of the population, future studies involving larger sample size are needed to confirm the present results and further elucidate the impact of $\mathrm{CHO}$ ingestion on cognitive performance specific to an intermittent sport model.

\section{Conclusions}

To summarize, the present data suggests that $\mathrm{CHO}$ ingestion enhances physical performance in high-level squash and fencing athletes during an intermittent high-intensity exercise, and has an additional positive effect on cognitive performances after exercise. Note that, the specificity of our high-level athletes can influence these results as it is generally assumed that attentional breadth continued to increase with higher physical exercise in contrast with non-athletes [53].

Acknowledgments: We thank all the subjects for participating to this study. Thank to Elliot Miller Henderson for the proofreading the final version of the manuscript.

Author Contributions: For research articles with several authors, a short paragraph specifying their individual contributions must be provided. The following statements should be used Jeanick Brisswalter conceived and designed the experiments; Laura Pomportes performed the experiments; Laura Pomportes 
and Jeanick Brisswalter analyzed the data; Arnaud Hays contributed reagents/materials/analysis tools; Laura Pomportes, Jeanick Brisswalter and Karen Davranche wrote the paper.

Conflicts of Interest: The authors declare no conflict of interest.

\section{References}

1. Gordon, B.; Kohn, L.A.; Levine, S.A.; Matton, M.; Scriver, W.D.M.; Whiting, W.B. Sugar content of the blood in runners following a marathon race. J. Am. Med. Assoc. 1925, 85, 508-509. [CrossRef]

2. Jeukendrup, A.E.; Rollo, I.; Carter, J.M. Carbohydrate mouth rinse: Performance effects and mechanisms. Sports Sci. Exch. 2013, 26, 1-8.

3. Meeusen, R. Exercise, Nutrition and the Brain. Sports Med. 2014, 44, 47-56. [CrossRef] [PubMed]

4. Newsholme, E.A.; Acworth, I.N.; Blomstrand, E. Amino acids, brain neurotransmitters and a functional link between muscle and brain that is important in sustained exercise. Adv. Myochem. 1987, 1, 127-133.

5. Meeusen, R.; Watson, P.; Dvorak, J. The Brain and Fatigue: New Opportunities for Nutritional Interventions. J. Sports Sci. 2006, 24, 773-782. [CrossRef] [PubMed]

6. Jeukendrup, A.E. Carbohydrate intake during exercise and performance. Nutrition 2004, 20, 669-677. [CrossRef] [PubMed]

7. Phillips, S.M. Carbohydrate supplementation and prolonged intermittent high-intensity exercise in adolescents: Research findings, ethical issues and suggestions for the future. Sports Med. 2012, 42, 817-828. [CrossRef] [PubMed]

8. Baker, L.B.; Rollo, I.; Stein, K.W.; Jeukendrup, A.E. Acute Effects of Carbohydrate Supplementation on Intermittent Sports Performance. Nutrients 2015, 7, 5733-5763. [CrossRef] [PubMed]

9. Dorling, J.L.; Earnest, C.P. Effect of carbohydrate mouth rinsing on multiple sprint performance. J. Int. Soc. Sports Nutr. 2013, 10. [CrossRef] [PubMed]

10. Rollo, I.; Homewood, G.; Williams, C.; Carter, J.; Goosey-Tolfrey, V.L. The influence of carbohydrate mouth rinse on self-selected intermittent running performance. Int. J. Sport Nutr. Exerc. Metab. 2015, 25, 550-558. [CrossRef] [PubMed]

11. Gant, N.; Stinear, C.M.; Byblow, W.D. Carbohydrate in the mouth immediately facilitates motor output. Brain Res. 2010, 1350, 151-158. [CrossRef] [PubMed]

12. De Pauw, K.; Roelands, B.; Knaepen, K.; Polfliet, M.; Stiens, J.; Meeusen, R. Effects of caffeine and maltodextrin mouth rinsing on P300. J. Appl. Physiol. 2015, 118, 776-782. [CrossRef] [PubMed]

13. Chambers, E.S.; Bridge, M.W.; Jones, D.A. Carbohydrate sensing in the human mouth: Effects on exercise performance and brain activity. J. Physiol. 2009, 587, 1770-1794. [CrossRef] [PubMed]

14. Turner, C.E.; Byblow, W.D.; Stinear, C.M.; Gant, N. Carbohydrate in the mouth enhances activation of brain circuitry involved in motor performance and sensory perception. Appetite 2014, 80, 212-219. [CrossRef] [PubMed]

15. Ali, A.; Williams, C.; Nicholas, C.W.; Foskett, A. The influence of carbohydrate-electrolyte ingestion on soccer skill performance. Med. Sci. Sports Exerc. 2007, 39, 1969-1976. [CrossRef] [PubMed]

16. Lee, C.L.; Cheng, C.F.; Astorino, T.A.; Lee, C.J.; Huang, H.W.; Chang, W.D. Effects of carbohydrate combined with caffeine on repeated sprint cycling and agility performance in female athletes. Int. Soc. Sports Nutr. 2014, 1, 11-17. [CrossRef] [PubMed]

17. Welsh, R.S.; Davis, J.M.; Burke, J.R.; Williams, H.G. Carbohydrates and physical/mental performance during intermittent exercise to fatigue. Med. Sci. Sports Exerc. 2002, 34, 723-731. [CrossRef] [PubMed]

18. Winnick, J.J.; Davis, J.M.; Welsh, R.S.; Carmichael, M.D.; Murphy, E.A.; Blackmon, J.A. Carbohydrate feedings during team sport exercise preserve physical and cns function. Med. Sci. Sports Exerc. 2005, 37, 306-315. [CrossRef] [PubMed]

19. Delignières, D.; Brisswalter, J.; Legros, P. Influence of physical exercise on choice reaction time in sport experts: The mediating role of resource allocation. J. Hum. Mov. Stud. 1994, 27, 173-188.

20. Etnier, J.L.; Salazar, W.; Landers, D.M.; Petruzzello, S.J.; Han, M.; Nowell, P. The influence of physical fitness and exercise upon cognitive functioning: A meta- analysis. J. Sport Exerc. Psychol. 1997, 19, $249-277$. [CrossRef]

21. Brisswalter, J.; Collardeau, M.; René, A. Effects of acute physical exercise characteristics on cognitive performance. Sports Med. 2002, 32, 555-566. [CrossRef] [PubMed] 
22. Collardeau, M.; Brisswalter, J.; Vercruyssen, F.; Audiffren, M.; Goubault, C. Single and choice reaction time during prolonged exercise in trained subjects: Influence of carbohydrate availability. Eur. J. Appl. Physiol. 2001, 86, 150-156. [PubMed]

23. Dietrich, A.; Audiffren, M. The reticular-activating hypofrontality (RAH) model of acute exercise. Neurosci. Biobehav. Rev. 2011, 35, 1305-1325. [CrossRef] [PubMed]

24. Baker, L.B.; Nuccio, R.P.; Jeukendrup, A.E. Acute effects of dietary constituents on motor skill and cognitive performance in athletes. Nutr. Rev. 2014, 72, 790-802. [CrossRef] [PubMed]

25. Bottoms, L.M.; Hunter, A.M.; Golloway, S.D.R. Effects of carbohydrate ingestion on skill maintenance in squash players. Eur. J. Sport Sci. 2006, 6, 187-195. [CrossRef]

26. Lieberman, H.R.; Falco, C.M.; Slade, S.S. Carbohydrate administration during a day of sustained aerobic activity improves vigilance, as assessed by a novel ambulatory monitoring device, and mood. Am. J. Clin. Nutr. 2002, 76, 120-127. [PubMed]

27. Utter, A.C.; Kang, J.; Nieman, D.C.; Williams, F.; Robertson, R.J.; Henson, D.A.; Davis, J.M.; Butterworth, D.E. Effect of carbohydrate ingestion and hormonal responses on ratings of perceived exertion during prolonged cycling and running. Eur. J. Appl. Physiol. Occup. Physiol. 1999, 80, 92-99. [CrossRef] [PubMed]

28. Léger, L.; Boucher, R. An indirect continuous running multistage field test: The Université de Montréal track test. Can. J. Appl. Sport Sci. 1980, 5, 77-84. [PubMed]

29. Driller, M.W.; Christos, K.A.; Cecilia, M.S. The Reliability of a 30-s Sprint Test on the Wattbike Cycle Ergometer. Int. J. Sports Physiol. Perform. 2013, 8, 379-383. [CrossRef] [PubMed]

30. Hopkins, W.G.; Marshall, S.W.; Batterham, A.M.; Hanin, J. Progressive Statistics for Studies in Sports Medicine and Exercise Science. Med. Sci. Sports Exerc. 2009, 41, 3-13. [CrossRef] [PubMed]

31. Beaven, C.M.; Maulder, P.; Pooley, A.; Kilduff, L.; Cook, C. Effects of caffeine and carbohydrate mouth rinses on repeated sprint performance. Appl. Physiol. Nutr. Metab. 2013, 38, 633-637. [CrossRef] [PubMed]

32. Phillips, S.M.; Findlay, S.; Kavaliauskas, M.; Grant, M.C. The Influence of Serial Carbohydrate Mouth Rinsing on Power Output during a Cycle Sprint. J. Sports Sci. Med. 2014, 13, 252-258. [PubMed]

33. Lambourne, K.; Tomporowski, P. The effect of exercise-induced arousal on cognitive task performance: A meta-regression analysis. Brain Res. 2010, 134, 12-24. [CrossRef] [PubMed]

34. McMorris, T. History of research into the acute exercise-cognition interaction: A cognitive psychology approach. In Exercise-Cognition Interaction: Neuroscience Perspectives; McMorris, T., Ed.; Elsevier: New York, NY, USA, 2016; pp. 1-28.

35. Podolin, D.A.; Munger, P.A.; Mazzeo, R.S. Plasma catecholamine and lactate response during graded exercise with varied glycogen conditions. J. Appl. Physiol. 1991, 71, 1427-1433. [PubMed]

36. Chmura, J.; Nazar, K.; Kaciuba-Uscilko, H. Choice reaction time during graded exercise in relation to blood lactate and plasma catecholamine thresholds. Int. J. Sports Med. 1994, 15, 172-176. [CrossRef] [PubMed]

37. Davranche, K.; Brisswalter, J.; Radel, R. Where are the limits of the exercise intensity on cognitive control? J. Sport Health Sci. 2015, 4, 56-63. [CrossRef]

38. Schmit, C.; Davranche, K.; Easthope, C.S.; Colson, S.S.; Brisswalter, J.; Radel, R. Pushing to the limits: The dynamics of cognitive control during exhausting exercise. Neuropsychologia 2015, 68, 71-81. [CrossRef] [PubMed]

39. Cermak, N.M.; Van Loon, J.C. The Use of Carbohydrates during Exercise as an Ergogenic Aid. Sports Med. 2013, 43, 1139-1155. [CrossRef] [PubMed]

40. Carter, J.M.; Jeukendrup, A.E.; Jones, D.A. The effect of carbohydrate mouth rinse on 1-h cycle time trial performance. Med. Sci. Sports Exerc. 2004, 36, 2107-2111. [CrossRef] [PubMed]

41. Glade, M.J. Caffeine-Not just a stimulant. Nutrition 2010, 26, 932-938. [CrossRef] [PubMed]

42. Hogervorst, E.; Bandelow, S.; Schmitt, J.; Jentjens, R.; Oliveira, M.; Allgrove, J.; Carter, T.; Gleeson, M. Caffeine Improves Physical and Cognitive Performance during Exhausting Exercise. Med. Sci. Sports Exerc. 2008, 40, 1841-1851. [CrossRef] [PubMed]

43. Santos, V.G.; Santos, V.R.; Felippe, L.J.; Almeida, J.W., Jr.; Bertuzzi, R.; Kiss, M.A.; Lima-Silva, A.E. Caffeine reduces reaction time and improves performance in simulated-contest of taekwondo. Nutrients 2014, 6, 637-649. [CrossRef] [PubMed] 
44. Veasey, R.C.; Haskell-Ramsay, C.F.; Kennedy, D.O.; Wishart, K.; Maggini, S.; Fuchs, C.J.; Stevenson, E.J. The Effects of Supplementation with a Vitamin and Mineral Complex with Guaraná Prior to Fasted Exercise on Affect, Exertion, Cognitive Performance, and Substrate Metabolism: A Randomized Controlled Trial. Nutrients 2015, 7, 6109-6127. [CrossRef] [PubMed]

45. Pomportes, L.; Davranche, K.; Brisswalter, I.; Hays, A.; Brisswalter, J. Heart Rate Variability and Cognitive Function Following a Multi-Vitamin and Mineral Supplementation with Added Guarana (Paullinia cupana). Nutrients 2015, 7, 196-208. [CrossRef] [PubMed]

46. Coull, N.A.; Watkins, S.L.; Aldous, J.W.; Warren, L.K.; Chrismas, B.C.; Dascombe, B.; Mauger, A.R.; Abt, G.; Taylor, L. Effect of tyrosine ingestion on cognitive and physical performance utilising an intermittent soccer performance test (iSPT) in a warm environment. Eur. J. Appl. Physiol. 2015, 115, 373-386. [CrossRef] [PubMed]

47. Fernstrom, J.J. Branched-Chain Amino Acids and Brain Function. J. Nutr. 2005, 135, 1539-1546.

48. Thompson, C.; Wylie, L.J.; Fulford, J.; Kelly, J.; Black, M.I.; McDonagh, S.T.J.; Jeukendrup, A.E.; Vanhatalo, A.; Jones, A.M. Dietary nitrate improves sprint performance and cognitive function during prolonged intermittent exercise. Eur. J. Appl. Physiol. 2015, 115, 1825-1834. [CrossRef] [PubMed]

49. Beelen, M.; Berghuis, J.; Bonaparte, B.; Ballak, S.B.; Jeukendrup, A.E.; van Loon, L.J. Carbohydrate mouth rinsing in the fed state: Lack of enhancement of time-trial performance. Int. J. Sport Nutr. Exerc. Metab. 2009, 19, 400-409. [CrossRef] [PubMed]

50. Fares, E.J.; Kayser, B. Carbohydrate mouth rinse effects on exercise capacity in pre-and postprandial States. J. Nutr. Metab. 2011. [CrossRef] [PubMed]

51. Whitham, M.; McKinney, J. Effect of a carbohydrate mouthwash on running time-trial performance. J. Sports Sci. 2007, 25, 1385-1392. [CrossRef] [PubMed]

52. Pottier, A.; Bouckaert, J.; Gilis, W.; Roels, T.; Derave, W. Mouth rinse but not ingestion of a carbohydrate solution improves 1-h cycle time trial performance. Med. Sci. Sports 2010, 20, 105-111. [CrossRef] [PubMed]

53. Hüttermann, S.; Memmert, D. Does the inverted-U function disappear in expert athletes? An analyses of attentional behavior under physical exercise of athletes and non-athletes. Physiol. Behav. 2014, 131, 87-92. [CrossRef] [PubMed]

(c) 2016 by the authors; licensee MDPI, Basel, Switzerland. This article is an open access article distributed under the terms and conditions of the Creative Commons Attribution (CC-BY) license (http://creativecommons.org/licenses/by/4.0/). 